\title{
Surface Plasmon Resonance Biosensor for Sensitive Detection of HER2-positive Exosomes Based on Reformative Tyramine Signal Amplification Activated by Molecular Aptamer Beacon Conversion
}

\section{Wenqin Chen}

Chongqing Medical University

\section{Zhiyang Li}

Nanjing Drum Tower Hospital: Nanjing University Medical School Affiliated Nanjing Drum Tower Hospital

\section{Wenqian Cheng}

Chongqing Medical University

\section{Tao Wu}

Zigong Fourth People's Hospital

Jia Li

Chongqing Medical University

\section{Xinyu Li}

Chongqing Medical University

Lin Liu

Zigong Fourth People's Hospital

Huijie Bai

Chongqing Medical University

\section{Shijia Ding}

Chongqing Medical University

\section{Xinmin Li}

Chongqing Medical University

Xiaolin Yu ( $\nabla$ yuxiaolincq@hotmail.com )

Zigong Fourth People's Hospital

\section{Research}

Keywords: HER2-positive exosomes, Surface plasmon resonance, Tyramine signal amplification, G4hemin, Breast cancer diagnosis 
Posted Date: October 25th, 2021

DOl: https://doi.org/10.21203/rs.3.rs-985834/v1

License: (c) (i) This work is licensed under a Creative Commons Attribution 4.0 International License. Read Full License 


\section{Abstract}

HER2-positive exosomes play an extremely important role in the diagnosis and treatment options of breast cancers. Herein, based on the reformative tyramine signal amplification (TSA) enabled by molecular aptamer beacon (MAB) conversion, a label-free surface plasmon resonance (SPR) biosensor was proposed for highly sensitive and specific detection of HER2-positive exosomes. The exosomes were captured by the HER2 aptamer region of MAB immobilized on the chip surface, which enabled the exposure of the G4 DNA that could form peroxidase-like G4-hemin. In turn, the formed G4-hemin catalyzed the deposition of plentiful tyramine-coated AuNPs (AuNPs-Ty) on the exosome membrane with the help of $\mathrm{H}_{2} \mathrm{O}_{2}$, generating significantly enhanced SPR signal. In the reformative TSA system, the horseradish peroxidase (HRP) as a major component was replaced with nonenzymic G4-hemin, bypassing the defects of nature enzymes. Moreover, the dual-recognition of the surface proteins and lipid membrane of the desired exosomes endowed the sensing strategy with high specificity without the interruption of free proteins. As a result, this developed SPR biosensor exhibited a wide linear range from $1.0 \times 10^{4}$ to $1.0 \times 10^{7}$ particles $/ \mathrm{mL}$. Importantly, this strategy was able to accurately distinguish HER2positive breast cancer patients from healthy individuals, exhibiting great potential clinical application.

\section{Introduction}

Breast cancer is the most common type of cancer that causes female deaths worldwide. The number of new breast cancer cases reached 2.26 million in 2020, surpassing lung cancer ( 2.21 million cases) for the first time to become the world's largest cancer $[1,2]$. In the diagnosis and treatment process of breast cancer, human epidermal growth factor receptor 2 (HER2) is an important prognostic indicator and predictor of HER2 targeted drugs [3, 4]. Currently, the clinical routine methods for HER2 analysis mainly include immunohistochemistry and fluorescence in situ hybridization, but these methods have several shortcomings, such as the uncertainty of the results, the high false-positive rates, and the excessively harsh requirements for specimens, etc [5]. Therefore, developing a noninvasive and reliable strategy to accurately monitor HER2 status is challenging but urgently needed.

Exosomes are 30-150 nm sized extracellular vesicles with a typical lipid bilayer membrane structure, which are secreted into the surrounding biofluids by different types of cells [6]. Through transporting various biological constituents of parent cells (lipids, nucleic acids and metabolites) into recipient cells, exosomes take part in cell-cell communication and are closely related to the occurrence and progress of disease [7]. In particular, tumor-derived exosomes not only carry cancer-specific proteins, but have high abundance and stability in body fluids, thus acting as promising biomarkers for liquid biopsy [8]. Recent reports have proved that the HER2-positive exosomes are greatly increased in patients with breast cancer, and there is the well consistency of the HER2 expression level between exosomes and tumor tissues [9, 10]. Therefore, quantitating the amounts of HER2-positive exosomes in clinical samples has the potential to improve the diagnosis and customize treatment of breast cancer. 
So far, apart from traditional methods including nanoparticle tracking analysis, western blot, flow cytometry, and enzyme-linked immunosorbent assay [11], various biosensing platforms have been developed for exosomes analysis by targeting their surface proteins using the corresponding antibodies or aptamers [12-17]. Among them, surface plasma resonance (SPR) biosensor is receiving extensive attention because it is a rapid, real-time, and label-free diagnostic device $[18,19]$. The sensing principle of this technique is to measure the change in refractive index around the surface of the metallic sensor chip due to alterations in mass caused by analyte-receptor noncovalent interactions [20,21]. However, SPR biosensor for exosomes detection faces adverse conditions. For instance, the small size and low mass of exosomes cannot trigger obvious signal discrepancy, leading to the inevitable requirement of signal amplifier. Moreover, the collected exosomes are always mingled with free target proteins from serum, resulting in the generation of false-positive signal that reduces the accuracy of results. Considering these facts, we are spurred to develop a novel SPR biosensing strategy for sensitive and specific detection of HER2-positive exosomes.

Tyramine signal amplification (TSA) is a typical enzyme-assisted amplification strategy in which tyramine or reporter molecules-functionalized tyramine can be transformed by horseradish peroxidase (HRP) into a reactive oxidized intermediate with the help of $\mathrm{H}_{2} \mathrm{O}_{2}$ [22]. The intermediate then covalently conjugates and rapidly deposits on near protein residues to trigger signal enhancement [23]. In previous studies, Jung et al. developed a TSA-based surface-enhanced Raman spectroscopy sensor by modifying gold nanoparticles (AuNPs) with tyramine and Raman-active probe, exhibiting significantly enhanced sensitivity [24]. Our group proposed a cytosensor that used HRP to catalyze the deposition of tyraminemodified electroactive reporters on tumor cells [25]. Nevertheless, HRP has been suffered from complicated purification processes and poor chemical stability due to the intrinsic characters of nature enzymes [26], which compromise the clinical applicability of TSA-based strategies. G-quadruplex-hemin (G4-hemin), an HRP-mimicking DNAzyme with excellent catalytic performance, has been widely applied in the biosensing owing to its easy synthesis, high stability, and low requirement for reaction conditions [27, 28]. Therefore, G4-hemin could be a credible and promising alternative to HRP.

Herein, a label-free SPR biosensor was proposed for highly sensitive and specific detection of HER2positive exosomes based on reformative TSA activated by target-induced molecular aptamer beacon (MAB) conversion. By combining the functions of G4 and HER2 aptamer into one molecular beacon, the engineered MABs immobilized on the sensing chip bound to HER2-positive exosomes, resulting in the structural change that accompanied the exposure of G4. Then, the formed G4-hemin catalyzed the deposition of numerous tyramine-coated AuNPs (AuNPs-Ty) on the lipid membrane of the exosomes in the presence of $\mathrm{H}_{2} \mathrm{O}_{2}$, achieving geometrically enhanced SPR signal. Profiting from the superior catalytic ability of G4-hemin, the improved TSA had no need for HRP. What's more, the dual-identification of surface proteins and the membrane structure of exosomes was able to avoid the interference of the free proteins. Taken together, the developed SPR biosensing strategy enabled accurate detection of HER2positive exosomes with high sensitivity and specificity. 


\section{Experimental}

\subsection{Reagents and materials}

Sodium citrate powder, $\mathrm{HAuCl}_{4} \cdot 4 \mathrm{H}_{2} \mathrm{O}$ and 11 -mercaptoundecanoic acid (MUA) were purchased from Sinopharm Chem Co., Ltd (Shanghai, China). Hemin, tyramine, and 6-Mercapto-1-hexanol (MCH) were purchased from Sigma-Aldrich (St. Louis, USA). Hemin was dissolved in dimethyl sulfoxide as the stock solution and then diluted to the required concentration with 25 mM HEPES buffer ( $\mathrm{pH}$ 7.4, 25 mM HEPES, $200 \mathrm{mM} \mathrm{NaCl}, 100 \mathrm{mM} \mathrm{KCl}, 1 \%$ DMSO, 0.05\% Triton). Fetal Bovine Serum (FBS) and Dulbecco's Modified Eagle Medium (DMEM) were offered by Gibco (Gaithersburg, USA). N-hydroxysuccinimide (NHS) and N(3-(dimethylamino) propyl)-N'-ethylcarbodiimide hydrochloride (EDC) were purchased from Alfa Aesar (Massachusetts, USA). Phosphate buffer (PBS) was supplied by Thermo Fisher Scientific (Wilmington, USA). All the chemicals used were of analytical reagent grade, and all the HPLC-purified oligonucleotides listed in Table S1 were prepared by Sangon Biotech. Co., Ltd (Shanghai, China). Tris-EDTA (TE) buffer (10 $\mathrm{mM}$ Tris-HCl, 1 mM EDTA, pH 8.0) was utilized for the dissolution of oligonucleotides. Aqueous solutions prepared by Millipore Milli-Q gradient ultrapure water system were used (Millipore Co., MA, USA).

\subsection{Instruments}

All measurements were carried out on the SPR biochemical analyzer independently developed and built by our own group. The SPR analyzer is based on the principles of physical optics and mainly composed of light source, a sensing chip, flow cell, and a CCD detector. The sensorgrams are analyzed by the labdeveloped program written in LabVIEW, exhibiting a time course of resonance units (RU). Transmission electron microscopic (TEM) image was performed with $\mathrm{H}-7500$ transmission electron microscope (Hitachi High-Technologies Co., Japan). Nanoparticles tracking analysis (NTA) was supported by ZetaView (Particle Metrix, Germany). UV-Visible absorption spectra were carried out at a UV-2550 spectrophotometer (Shimadzu, Japan).

\subsection{Extraction of cancer cell-derived exosomes}

All cell lines, including HER2-positive SK-BR3 and HER2-negative HeLa, LNCaP, HepG2, and MCF-7 [2932], were provided by the American Type Culture Collection (ATCC) (Rockville, USA). DMEM (added with $10 \%$ FBS, $1 \%$ streptomycin and penicillin) was used for all kinds of cell culture in sterile incubator containing $5 \% \mathrm{CO}_{2}$, at $37{ }^{\circ} \mathrm{C}$. Cell passaging was processed when growth density reached $80 \%$. Ultimately, the cells were starved with serum-free medium for $48 \mathrm{~h}$ before the exosomes were extracted.

The exosomes were extracted from the cell culture medium according to the published literatures [33, 34]. Generally, after 48 h's starvation, the cell culture medium was centrifuged $(2,000 \times \mathrm{g}, 20 \mathrm{~min} ; 10,000 \times \mathrm{g}$, $30 \mathrm{~min} ; 100,000 \times \mathrm{g}, 2 \mathrm{~h}$. All steps were performed at $\left.4{ }^{\circ} \mathrm{C}\right)$. The precipitate containing exosomes was suspended by $1 \times$ PBS and characterized by TEM and NTA. Clinical samples were collected from the First Affiliated Hospital of Chongqing Medical University. And the extraction procedures of exosomes from clinical samples were as described above. 


\subsection{Preparation of AuNPs-Ty complex}

Firstly, AuNPs with a particle size of about $14 \mathrm{~nm}$ were synthesized according to the published literatures with slight modification $[35,36]$. Briefly, $88.2 \mathrm{~mL}$ of $\mathrm{HAuCl}_{4}(1 \mathrm{mM})$ was boiled and then added rapidly to $10.1 \mathrm{~mL}$ of sodium citrate (34 mM). After the color changed to burgundy, the mixture was stopped heating and slowly cooled to room temperature with continued stirring. Then the prepared AuNPs and tyramine were combined by MUA that has sulfhydryl and carboxyl groups at each end. In detail, the sulfhydryl groups at one end of MUA was connected to AuNPs through Au-S bonds, and the other end was connected to tyramine through carboxyl groups, which indirectly connected AuNPs and tyramine into AuNPs-Ty complex. To begin with, add MUA $(25 \mu \mathrm{L}, 100 \mu \mathrm{M})$ to the AuNPs $(5 \mathrm{~mL})$ solution, gently shaking for 12 h's reaction, then centrifuge the solution twice $(2,000 \times \mathrm{g}, 10 \mathrm{~min})$ to get AuNPs-MUA solution. Thereafter, add NHS (10 $\mu \mathrm{L}, 1 \mathrm{mg} / \mathrm{mL}), \mathrm{EDC}(10 \mu \mathrm{L}, 2 \mathrm{mg} / \mathrm{mL})$ and tyramine $(10 \mu \mathrm{L}, 100 \mu \mathrm{M})$ to the mixed solution, gently shaking for 12 h's reaction to get AuNPs-Ty complex, stored at $4{ }^{\circ} \mathrm{C}$ for later use.

\subsection{Exosomes analysis by the fabricated SPR biosensor}

Firstly, immerse the gold chip and the flow cell in 75\% ethanol, sonicate for $20 \mathrm{~min}$ at room temperature. Rinse with deionized water and blow dry with nitrogen. Then, freshly prepare the piranha solution $1 \mathrm{~mL}$ $\left(\mathrm{H}_{2} \mathrm{SO}_{4}: 700 \mu \mathrm{L}, \mathrm{H}_{2} \mathrm{O}_{2} 300 \mu \mathrm{L}\right)$, after cooling to room temperature, cover the chip surface for $10 \mathrm{~min}$ to remove the remaining impurities. Rinse the chip thoroughly with deionized water and blow dry with nitrogen. Next, dilute the capture probes of MAB to a concentration of $1.5 \mu \mathrm{M}$ by the fixative solution $\left(\mathrm{KH}_{2} \mathrm{PO}_{4}, 1 \mathrm{M}\right)$, modified on the surface of the chip overnight at $4{ }^{\circ} \mathrm{C}$, and followed by the block of $1 \mathrm{mM}$ $\mathrm{MCH}$ for $30 \mathrm{~min}$. After wash and dry the chip, install the treated chip, the prism cleaned with $75 \%$ ethanol, and the flow cell on the SPR instrument for subsequent testing. Rinse the flow pipeline with cell grade $1 \times$ PBS for 15 min to output the baseline signal (flow rate: $50 \mu \mathrm{L} / \mathrm{min}$ ). Then, different concentrations of exosomes derived from tumor cells or clinical samples were loaded (loading volume: $150 \mu \mathrm{L}$, flow rate: 7 $\mu \mathrm{L} / \mathrm{min}$, time: $30 \mathrm{~min}$ ). Subsequently, loading the AuNPs-Ty composite (loading volume: $150 \mu \mathrm{L}$, flow rate: $7 \mu \mathrm{L} / \mathrm{min}$, time: $30 \mathrm{~min})$. After detection, the surface of the sensing chip was regenerated with $50 \mathrm{mM}$ $\mathrm{NaOH}$ (loading volume: $150 \mu \mathrm{L}$, flow rate: $20 \mu \mathrm{L} / \mathrm{min}$, time: $2 \mathrm{~min}$ ).

\section{Results And Discussion}

\subsection{Illustration of the SPR biosensing strategy}

Scheme 1. Schematic illustration for the detection of HER2-positive exosomes based on the improved TSA enabled by target-induced MAB conversion.

\subsection{Characterization of the extracted exosomes, G4-hemin, and AuNPs-Ty}

First, the morphology and concentration of exosomes derived from SK-BR3 cells were characterized, and the results were shown in Fig. S1. The TEM image disclosed that the exosomes were round vesicles with 
a clear double-layer membrane structure (Fig. S1A). The NTA indicated that the original concentration of the exosomes was $2.75 \times 10^{9}$ particles $/ \mathrm{mL}$ with a size distribution ranging from 30 to $200 \mathrm{~nm}$ (Fig. S1B). The above results confirmed the successful extraction of exosomes. Second, the combination of G4 and hemin was verified by UV-vis absorption spectroscopy. As we can learn from Fig. S2, $260 \mathrm{~nm}$ and $394 \mathrm{~nm}$ were the characteristic absorption peaks of G4 and hemin, respectively. As expected, in the absorption spectrum of G4-hemin, the absorption peaks of the two substances with a slight right shift were observed at the same time, indicating the successful formation of G4-hemin. Third, the characterization of AuNPsTy composite was performed. The TEM image showed the prepared AuNPs were uniform in volume and evenly distributed (Fig. 1A), and the UV-vis absorption spectra displayed that the typic absorption peaks of AuNPs and AuNPs-Ty were seen at $530 \mathrm{~nm}$ and $537 \mathrm{~nm}$, respectively (Fig. 1B). These results were entirely consistent with the previous report [24], indicating the successful synthesis of AuNPs-Ty.

\subsection{Feasibility of the developed biosensor}

To demonstrate the feasibility of this proposed biosensor, the whole detection process was firstly analyzed. The detection steps and the corresponding sensorgrams were depicted in Fig. 2A and B, respectively. In the first step, when the HER2-positive exosomes were loaded, the SPR signal rose by about $40 \mathrm{RU}$, indicating that the exosomes were connected to the surface of the chip owing to the effective binding of the aptamer of the MAB and HER2 proteins. In the second step, once the loading of AuNPs-Ty composite, the SPR signal increased by about $200 \mathrm{RU}$, which was attributed to the deposition of plentiful AuNPs-Ty on the surface of the exosomal membrane caused by the reformative TSA. After detection, the chip surface was regenerated with $50 \mathrm{mM} \mathrm{NaOH}$ for next measurement. Subsequently, to verify that the deposition of AuNPs-Ty on the exosome surface was caused by the catalytic activity of G4-hemin rather than the nonspecific absorption, the relevant experiments were conducted. As shown in Fig. $2 \mathrm{C}$, in the absence of hemin, the signal of the AuNPs-Ty was not been observed due to the fact that the exposed G4 had not peroxidase-like activity. In contrast, the AuNPs-Ty generated distinct SPR signal, demonstrating that the signal indeed originated from the reformative TSA. In addition, compared with Ty and AuNPs that produced negligible SPR signal, the signal of AuNPs-Ty increased nearly 10-fold (Fig. 2D). These results adequately demonstrated the feasibility of the proposed method for the detection of HER2-positive exosomes.

\subsection{Optimization of experimental parameters}

Several important reaction parameters of this biosensor were optimized to obtain good analytical performance. Owing to the effect of the density of capture probe immobilized on sensing chip on the sensitivity of the biosensor, the concentration of the MAB $\left(C_{M A B}\right)$ was firstly optimized. As shown in Fig. $S 3 A$, the SPR signal gradually improved with the increase of the $C_{M A B}$ ranging from 0.5 to $1.5 \mu \mathrm{M}$. Once the concentration was above $1.5 \mu \mathrm{M}$, the signal decreased rapidly, because the crowded MAB could not bind to the exosomes effectively. Therefore, $1.5 \mu \mathrm{M}$ was chosen as the optimal $\mathrm{C}_{\mathrm{MAB}}$. When the 
concentration of hemin $\left(C_{\text {hemin }}\right)$ was $1.5 \mu \mathrm{M}$, being same as the $C_{M A B}$, the highest signal was obtained. Hence, the optimal $C_{\text {hemin }}$ is set to $1.5 \mu \mathrm{M}$ (Fig. S3B).

Furthermore, to avoid the nonspecific deposition of redundant signal amplifier on the chip surface which increased the background signal, the befitting concentration of the AuNPs-Ty $\left(\mathrm{C}_{\mathrm{AuNPs}-\mathrm{Ty}}\right)$ was evaluated. As shown in Fig. S3C, when the $C_{\text {AuNPs-Ty }}$ rose from 50 to 200 mM, the SPR signal rose synchronously and ended by a downward trend. Therefore, we chose $200 \mathrm{mM}$ as the optimal $\mathrm{C}_{\text {AuNPs-Ty. Subsequently, }}$ we also optimized the reaction time between exosomes and MAB ( $\left.\mathrm{Time}_{1}\right)$ and the incubation time of the improved TSA ( $\mathrm{Time}_{2}$ ), respectively (Fig. S3D and E). When Time ${ }_{1}$ was set as $30 \mathrm{~min}$, the signal reached the peak and remained steady as time prolonged, indicating the completion of the reaction. Similarly, in the optimization experiment of $\mathrm{Time}_{2}$, the reaction was completed after $30 \mathrm{~min}$. Therefore, we set both reaction times as $30 \mathrm{~min}$.

\subsection{Sensitivity of the SPR biosensor}

After gaining the optimal reaction conditions, we further evaluated the sensitivity of this sensing method by detecting a series of different concentrations of HER2-positive exosomes. As shown in Fig. 3A, when the concentration of exosomes increased from 0.1 to $100 \times 10^{5}$ particles $/ \mathrm{mL}$, the SPR signal also increased simultaneously, showing a good correlation. As presented in, the regression equation between the concentration of exosomes $(X)$ and the corresponding SPR signals $(Y)$ was $Y=2.36 X+18.93$ with correlation coefficient of 0.9914 (Fig. 3B). The lowest detectable concentration of the sensing strategy was $1.0 \times 10^{4}$ particles $/ \mathrm{mL}$, which was comparable to other biosensing strategies for exosomes detection, and the detailed results were shown in Table S2. The excellent analytical performance of this method is mainly due to these aspects. First, the high affinity of the aptamer to HER2 proteins allowed the G4 sequence to be fully exposed to form the G4-hemin. Second, the formed G4-hemin exerted superior peroxidase-like activity itself to catalytic the in situ deposition of numerous AuNPs-Ty on the exosomes, resulting the high SPR signal.

\subsection{Specificity of the SPR biosensing strategy}

The good specificity is one of the indispensable characteristics of an excellent biosensor, which directly affects the clinical application prospects of this method. Accordingly, we conducted follow-up researches to explore the specificity of this sensing strategy by choosing another four kinds of exosomes derived from different HER2-negative cancer cells (HeLa, LNcap, HepG2, and MCF-7) as interferents. As shown in Fig. 4, compared with these interferents, exosomes from HER2-positive SK-BR3 cells produced a significantly improved SPR signal. In general, the SPR signal of HER2-positive exosomes was 4.9 to 6.4 times to those of interfering substances at the same concentration $\left(1.0 \times 10^{7}\right.$ particles $\left./ \mathrm{mL}\right)$. Besides, by detecting exosomes using the developed strategy, the cancer cells with high HER2 expression could be discriminated accurately. These results showed the excellent specificity of this method, which was mainly attributed to the dual-identification of HER2 proteins and the lipid membrane of exosomes. 


\subsection{Detection of exosomes in clinical samples}

In order to evaluate the applicability of this method for the diagnosis of breast cancer, exosomes derived from real clinical samples were analyzed. We collected 16 serum samples from the First Affiliated Hospital of Chongqing Medical University. Among them, half of the samples were from HER2-positive breast cancer patients (P1 P8), and the rest were from healthy groups $(\mathrm{H} 1 \sim \mathrm{H} 8)$, which served as testing control. As shown in Fig. 5A, almost all SPR signals obtained by detecting exosomes from the serum of HER2-positive breast cancer patients were greater than $150 \mathrm{RU}$ (except for P2), however, under the same circumstances, the signals obtained by detecting exosomes from healthy samples were all lower than 50 $\mathrm{RU}$. In addition, the calculation of the significance probability $\mathrm{P}$ value also illustrated the significant difference between the two groups of data (Fig. 5B), implying the outstanding distinction ability of this method between HER2-positive breast cancer patients and healthy groups. These results shed light on the potential clinical application prospect of this sensing method.

\section{Conclusions}

In conclusion, a label-free SPR biosensor has been developed for highly sensitive and specific detection of HER2-positive exosomes based on the reformative TSA activated by target-induced MAB conversion. Different from the traditional TSA, the reformative TSA replacing HRP with G4-hemin fully avoids the intrinsic disadvantages of nature enzymes, thus possessing high potential for clinical transformation. And the biosensing strategy is able to simultaneously recognize HER2 proteins and the lipid membrane of the exosomes, which effectively eliminates the interference of free proteins in purified exosomes.

Benefiting from the integrating of the MAB and the reformative TSA, large quantities of AuNPs-Ty are insitu deposited on the HER2-positive exosome membrane, endowing the developed SPR biosensor with high sensitivity and specificity. Moreover, by simply changing the aptamer types, this sensing strategy can be easily extended for accurate detection of other exosome subtypes. More importantly, this method has capable of discerning patients with HER2-positive breast cancer from healthy individuals. Overall, this work offers a new SPR platform for exosome-based liquid biopsy in the diagnosis of breast cancer. Despite these results, a limitation of this method is that the experimental steps need to be further simplified to improve the flexibility in clinical applications.

\section{Abbreviations}

TSA

Tyramine signal amplification

MAB

Molecular aptamer beacon

SPR

Surface plasmon resonance

AuNPs-Ty

Tyramine-coated AuNPs 
HRP

Horseradish peroxidase

HER2

Human epidermal growth factor receptor 2

AuNPs

Gold nanoparticles

G4-hemin

G-quadruplex-hemin, MCH:6-Mercapto-1-hexanol

FBS

Fetal Bovine Serum

DMEM

Dulbecco's Modified Eagle Medium

NHS

$\mathrm{N}$-hydroxysuccinimide

EDC

$\mathrm{N}$-(3-(dimethylamino) propyl)-N'-ethylcarbodiimide hydrochloride

PBS

Phosphate buffer

$\mathrm{RU}$

Resonance units

TEM

Transmission electron microscopic

NTA

Nanoparticles tracking analysis.

\section{Declarations}

\section{Authors' contributions}

W. Chen, Z. Li, and W. Cheng performed all experimental work. J. Li, X. Li, and L. Liu conducted data analysis. T. Wu and H. Bai collected clinical samples. S. Ding and X. Li revised the manuscript and provided project guidance. X. Yu procured funding and data curation. All authors read and approved the final manuscript.

\section{Acknowledgements}

This work was supported by the National Natural Science Foundation of China (81873980), the National Science and Technology Major Project of the Ministry of Science and Technology of China (2018ZX10732202), and the Science and Technology Project of the Health Planning Committee of Sichuan (19PJ159).

\section{Availability of data and materials}


All data generated and analyzed during this study are included in this article and additional file. The additional file is available. Characterization of the exosomes, characterization of G4-hemin, and Optimizations of reaction conditions (Additional file: Figures S1-S3). Sequences of oligonucleotides employed in this work (Table S1). Comparison of biosensing strategies for the detection of exosomes (Table S2).

\section{Ethics approval and consent to participate}

This study has been approved by the ethics committee of Chongqing Medical University and conducted in accordance with ethical guidelines.

\section{Consent for publication}

All authors have provided consent for the manuscript to be published.

\section{Competing interests}

The authors declare that they have no competing interests.

\section{References}

[1] E. Heer, A. Harper, N. Escandor, H. Sung, V. McCormack, M.M. Fidler-Benaoudia, Global burden and trends in premenopausal and postmenopausal breast cancer: a population-based study, Lancet Glob. Health 8 (2020) e1027-e1037.

[2] Y. Li, Q. Li, H. Mo, X. Guan, S. Lin, Z. Wang, Y. Chen, Y. Zhang, D. Zhang, S. Chen, R. Cai, J. Wang, Y. Luo, Y. Fan, P. Yuan, P. Zhang, Q. Li, F. Ma, B. Xu, Incidence, risk factors and survival of patients with brain metastases at initial metastatic breast cancer diagnosis in China, Breast 55 (2021) 30-36.

[3] M. Mitsogianni, I.P. Trontzas, G. Gomatou, S. loannou, N.K. Syrigos, E.A. Kotteas, The changing treatment of metastatic her2-positive breast cancer, Oncol. Lett. 21 (2021) 287.

[4] T.Y. Semiglazova, S.M. Sharashenidze, S.N. Kerimova, V.V. Klimenko, V.F. Semiglazova, Current approaches to the treatment of HER2positive breast cancer with brain metastases, Tumors of Female Reproductive System 17(2021) 27-34.

[5] E. Hassan, B. Narjiss, K. Mouna, A. Abdelkader, G. Meryem, Safety of a trastuzumab biosimilar, used under routine clinical practice conditions in adult her2+ breast cancer patients in morocco, J. Clin. Oncol. 38 (2020) e13024-e13024.

[6] Y. Zhang, J. Bi, J. Huang, Y. Tang, S. Du, P. Li, Exosome: A Review of Its Classification, Isolation Techniques, Storage, Diagnostic and Targeted Therapy Applications, Int. J. Nanomedicine 22 (2020) 6917-6934. 
[7] Y. Shi, L. Du, D. Lv, Y. Li, Z. Zhang, X. Huang, H. Tang, Emerging role and therapeutic application of exosome in hepatitis virus infection and associated diseases, J. Gastroenterol. 56 (2021) 336-349.

[8] C. Han, C. Zhang, H. Wang, L. Zhao, Exosome-mediated communication between tumor cells and tumor-associated macrophages: implications for tumor microenvironment, Oncoimmunology. 10 (2021) 1887552.

[9] S. Fang, H. Tian, X. Li, D. Jin, X. Li, J. Kong, C. Yang, X. Yang, Y. Lu, Y. Luo, B. Lin, W. Niu, T. Liu, Clinical application of a microfluidic chip for immunocapture and quantification of circulating exosomes to assist breast cancer diagnosis and molecular classification, PLoS One 12 (2017) e0175050.

[10] Y. Kabe, M. Suematsu, S. Sakamoto, M. Hirai, I. Koike, T. Hishiki, A. Matsuda, Y. Hasegawa, K. Tsujita, M. Ono, N. Minegishi, A. Hozawa, Y. Murakami, M. Kubo, M. Itonaga, H. Handa, Development of a Highly Sensitive Device for Counting the Number of Disease-Specific Exosomes in Human Sera, Clin. Chem. 64 (2018) 1463-1473.

[11] H. Yan, Y. Li, S. Cheng, Y. Zeng, Advances in Analytical Technologies for Extracellular Vesicles, Anal. Chem. 93 (2021) 4739-4774.

[12] A. Bagheri Hashkavayi, B.S. Cha, E.S. Lee, S. Kim, K.S. Park, Advances in Exosome Analysis Methods with an Emphasis on Electrochemistry, Anal. Chem. 92 (2020) 12733-12740.

[13] H.M. Kim, C. Oh, J. An, S. Baek, S. Bock, J. Kim, H.S. Jung, H. Song, J.W. Kim, A. Jo, D.E. Kim, W.Y. Rho, J.Y. Jang, G.J. Cheon, H.J. Im, B.H. Jun, Multi-Quantum Dots-Embedded Silica-Encapsulated Nanoparticle-Based Lateral Flow Assay for Highly Sensitive Exosome Detection, Nanomaterials 11 (2021) 768.

[14] D.J. Timson, Myosin Va and spermine synthase: partners in exosome transport, Biosci. Rep. 39 (2019) BSR20190326.

[15] Y. Zhang, Y. Wei, P. Liu, X. Zhang, Z. Xu, X. Tan, M. Chen, J. Wang, ICP-MS and Photothermal DualReadout Assay for Ultrasensitive and Point-of-Care Detection of Pancreatic Cancer Exosomes, Anal. Chem. 93 (2021) 11540-11546.

[16] X.W. Zhang, M.X. Liu, M.Q. He, S. Chen, Y.L. Yu, J.H. Wang, Integral Multielement Signals by DNAProgrammed UCNP-AuNP Nanosatellite Assemblies for Ultrasensitive ICP-MS Detection of Exosomal Proteins and Cancer Identification, Anal. Chem. 93 (2021) 6437-6445.

[17] J. Lim, M. Choi, H. Lee, Y.H. Kim, J.Y. Han, E.S. Lee, Y. Cho, Direct isolation and characterization of circulating exosomes from biological samples using magnetic nanowires. J Nanobiotechnology. 17 (2019) 1.

[18] W. Chen, J. Li, X. Wei, Y. Fan, H. Qian, S. Li, Y. Xiang, S. Ding, Surface plasmon resonance biosensor using hydrogel-AuNP supramolecular spheres for determination of prostate cancer-derived exosomes, 
Microchim. Acta. 187 (2020) 590.

[19] W. Wu, X. Yu, J. Wu, T. Wu, Y. Fan, W. Chen, M. Zhao, H. Wu, X. Li, S. Ding, Surface plasmon resonance imaging-based biosensor for multiplex and ultrasensitive detection of NSCLC-associated exosomal miRNAs using DNA programmed heterostructure of Au-on-Ag, Biosens. Bioelectron. 175 (2021) 112835.

[20] R. Bakhtiar, Surface plasmon resonance spectroscopy: a versatile technique in a biochemist's toolbox, J. Chem. Edu. 90 (2013) 203-209.

[21] J. Das, S.O. Kelley, High-Performance Nucleic Acid Sensors for Liquid Biopsy Applications, Angew. Chem. Int. Ed. Engl. 59 (2020) 2554-2564.

[22] J. Dopie, M.J. Sweredoski, A. Moradian, A.S. Belmont, Tyramide signal amplification mass spectrometry (TSA-MS) ratio identifies nuclear speckle proteins, J. Cell Biol. 219 (2020) e201910207.

[23] C. Zong, F. Jiang, X. Wang, P. Li, L. Xu, H. Yang, Imaging sensor array coupled with dual-signal amplification strategy for ultrasensitive chemiluminescence immunoassay of multiple mycotoxins, Biosens. Bioelectron. 177 (2021) 112998.

[24] C. Fu, S. Jin, W. Shi, J. Oh, H. Cao, Y.M. Jung, Catalyzed Deposition of Signal Reporter for Highly Sensitive Surface-Enhanced Raman Spectroscopy Immunoassay Based on Tyramine Signal Amplification Strategy, Anal. Chem. 90 (2018) 13159-13162.

[25] X. Zhou, Y. Li, H. Wu, W. Huang, H. Ju, S. Ding, A amperometric immunosensor for sensitive detection of circulating tumor cells using a tyramide signal amplification-based signal enhancement system, Biosens. Bioelectron. 130 (2019) 88-94.

[26] B.B. Kou, Y.Q. Chai, Y.L. Yuan, R. Yuan, A DNA nanopillar as a scaffold to regulate the ratio and distance of mimic enzymes for an efficient cascade catalytic platform, Chem. Sci. 12 (2020) 407-411.

[27] Li. Jia, T. Yuan, T. Yang, L. Xu, L. Zhang, Li. Huang, W. Cheng, S. Ding, DNA-grafted hemin with preferable catalytic properties than G-quadruplex/hemin for fluorescent miRNA biosensing, Sens. Actuators, B. 271 (2018) 239-246.

[28] V. Javan Kouzegaran, K. Farhadi, M. Forough, M. Bahram, Ö. Persil Çetinkol, Highly-sensitive and fast detection of human telomeric G-Quadruplex DNA based on a hemin-conjugated fluorescent metal-organic framework platform, Biosens. Bioelectron. 178 (2021) 112999.

[29] S.Y. Digehsaraei, M. Salouti, B. Amini, S. Mahmazi, M. Kalantari, A. Kazemizadeh, J. Mehrvand, Developing a fluorescence immunosensor for detection of her2-positive breast cancer based on graphene and magnetic nanoparticles, Microchem. J. 167 (2021) 106300

[30] H. Xu, Z.P. Aguilar, L. Yang, M. Kuang, H. Duan, Y. Xiong, H. Wei, A. Wang, Antibody conjugated magnetic iron oxide nanoparticles for cancer cell separation in fresh whole blood, Biomaterials 32 (2011) 
[31] C. Feng, L.Y. Zhou, T. Yu, G. Xu, H.L. Tian, J.J. Xu, H.X. Xu, K.Q. Luo, A new anticancer compound, oblongifolin $\mathrm{C}$, inhibits tumor growth and promotes apoptosis in HeLa cells through Bax activation, Int. J. Cancer. 131 (2012) 1445-1454.

[32] M. Dahl, P. Bouchelouche, G. Kramer-Marek, J. Capala, J. Nordling, K. Bouchelouche, Sarcosine induces increase in HER2/neu expression in androgen-dependent prostate cancer cells. Mol. Biol. Rep. 38 (2011) 4237-4243.

[33] J. Liu, L. Zhu, L. Wang, Y. Chen, B.R. Giri, J. Li, G. Cheng, Isolation and Characterization of Extracellular Vesicles from Adult Schistosoma japonicum, J. Vis. Exp. 22 (2018) 57514.

[34] M.J. Ramírez-Bajo, E. Banon-Maneus, J. Rovira, J.M. Campistol, F. Diekmann, Isolation of Extracellular Vesicles Derived from Mesenchymal Stromal Cells by Ultracentrifugation, Bio. Protoc. 10 (2020) e3860.

[35] R. Gen, M. Ortiz, C.K. O'Sullivan, Diffusion-controlled synthesis of gold nanoparticles: nano-liposomes as mass transfer barrier, J. Nanoparticle Res. 16 (2014) 1-5.

[36] P.D. Nguyen, X. Zhang, J. Su, One-Step Controlled Synthesis of Size-Tunable Toroidal Gold Particles for Biochemical Sensing, ACS Appl. Nano Mater. 2 (2019), 7839-7847.

\section{Scheme 1}

Scheme 1 is available in the Supplementary Files section.

\section{Figures}



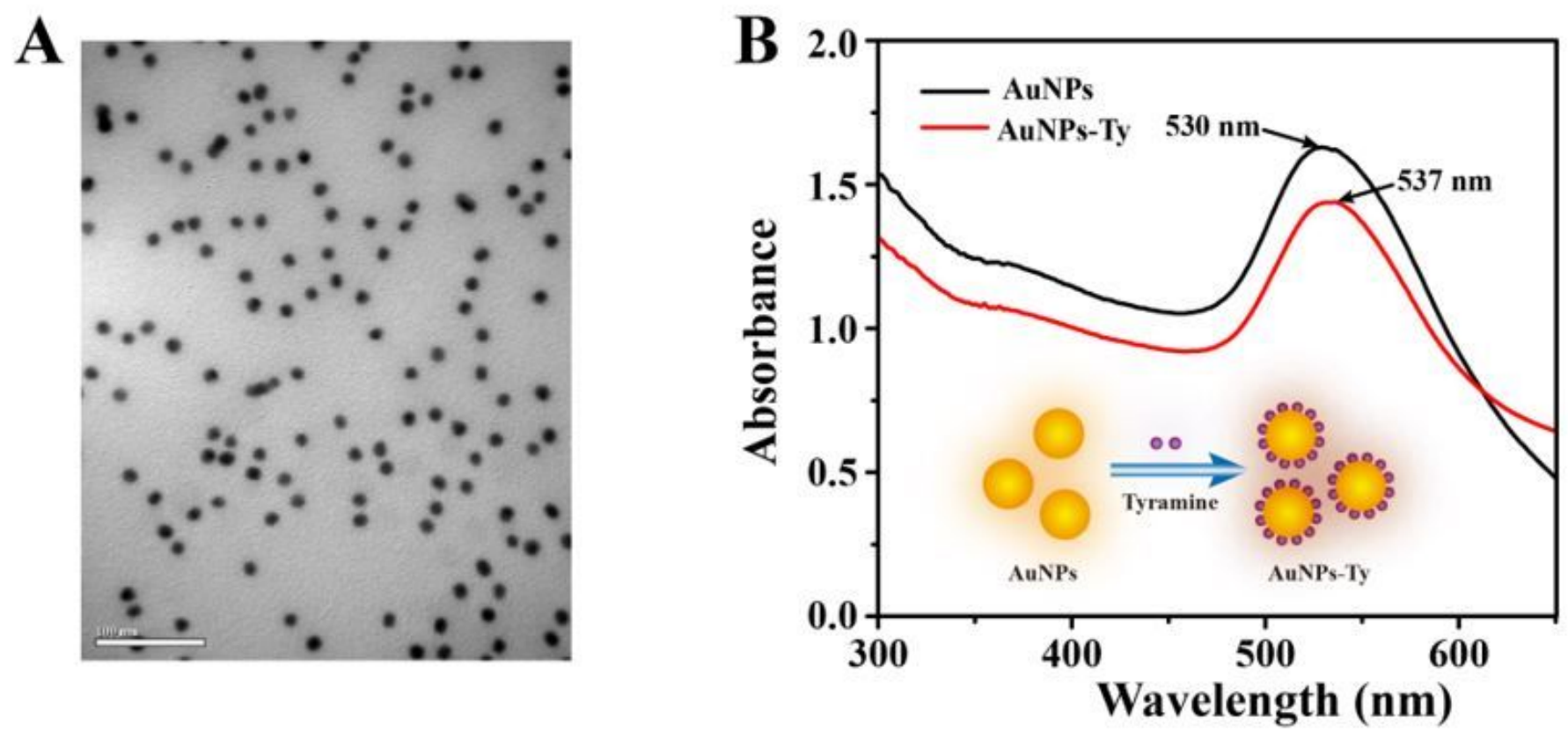

Figure 1

Characterization of the signal amplifier of AuNPs-Ty. (A) TEM image of AuNPs. Scale bars $=100 \mathrm{~nm}$, (B) UV-vis absorption spectra of AuNPs (black line) and AuNPs-Ty (red line). 
A
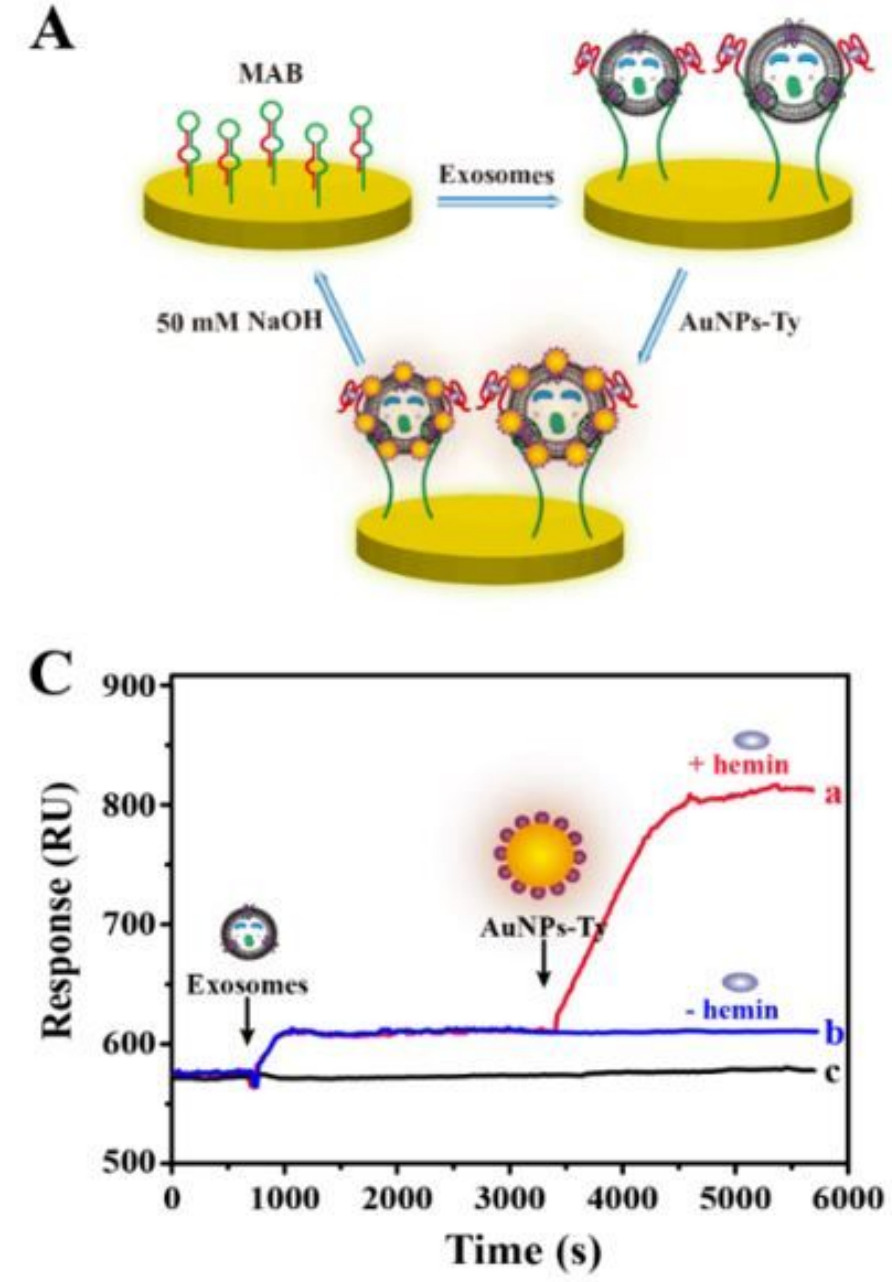
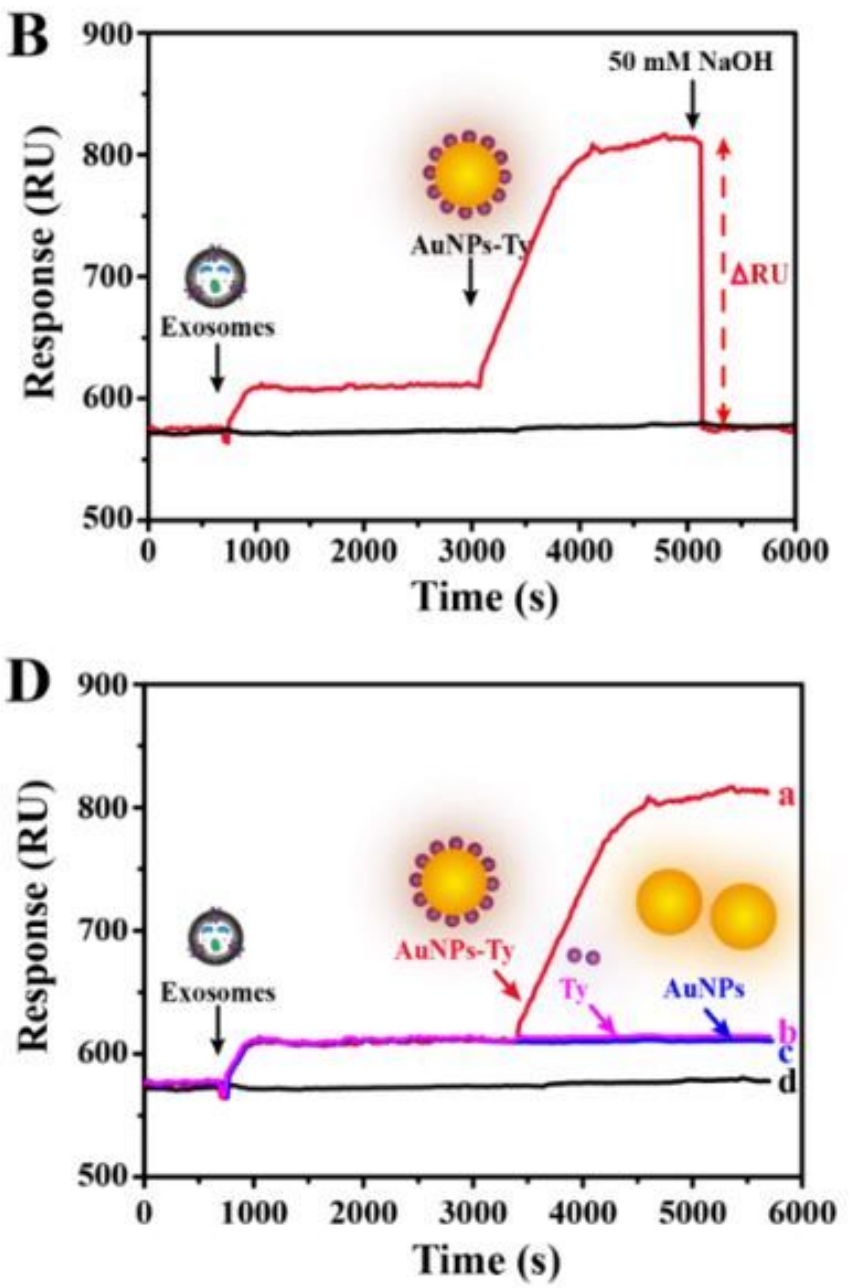

\section{Figure 2}

Evaluation of the feasibility of the biosensing strategy. (A) Schematic diagram of the detection process.

(B) Typical SPR sensorgrams of the biosensor (red line) and blank control without loading (black line). (C) SPR sensorgrams of the different conditions: (a) the intact sensing system, (b) the sensing system without hemin, and (c) blank control. (D) SPR sensorgrams of the different materials: (a) AuNPs-Ty, (b) Ty, (c) AuNPs, and (d) blank control. The concentration of HER2-positive exosomes derived from SK-BR3 cells is $1 \times 107$ particles $/ \mathrm{mL}$. All data expressed as mean \pm standard variation $(n=3)$. 

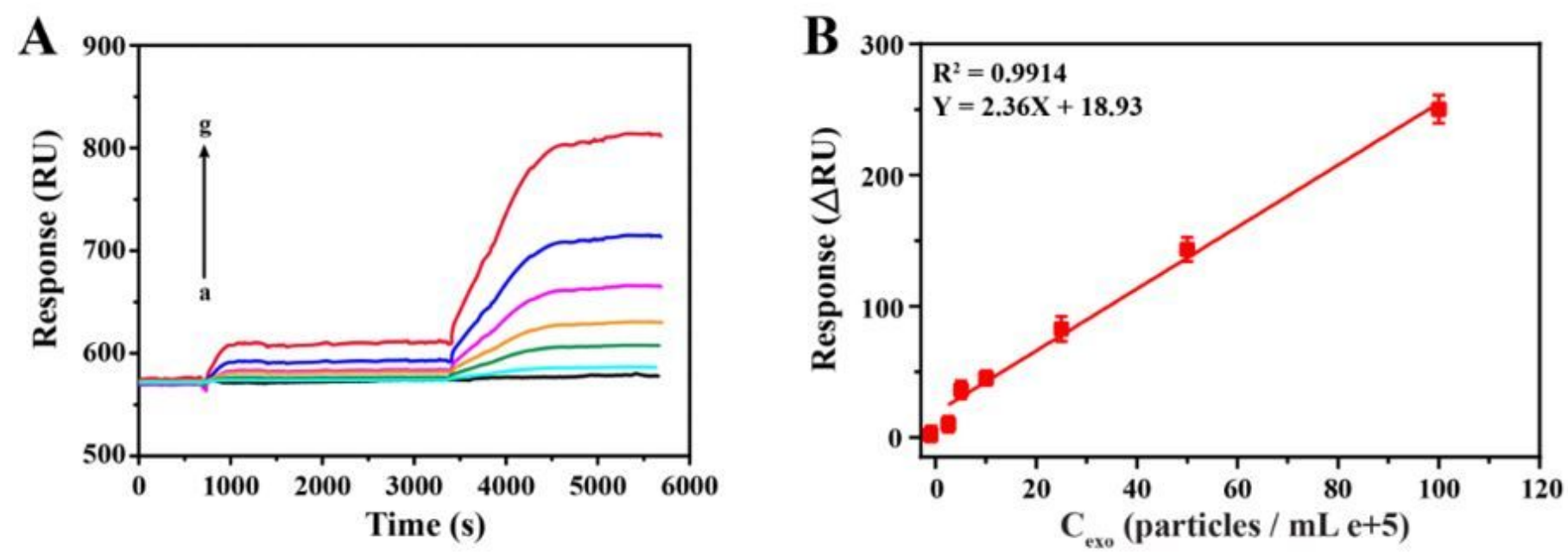

Figure 3

Evaluation of the sensitivity of the SPR biosensor. (A) SPR sensorgrams and (B) the calibration line for exosomes at different concentrations of $0.1,2.5,5,10,25,50,100$ particles/mL e+5 (from a to g). All data expressed as mean \pm standard variation $(n=3)$.
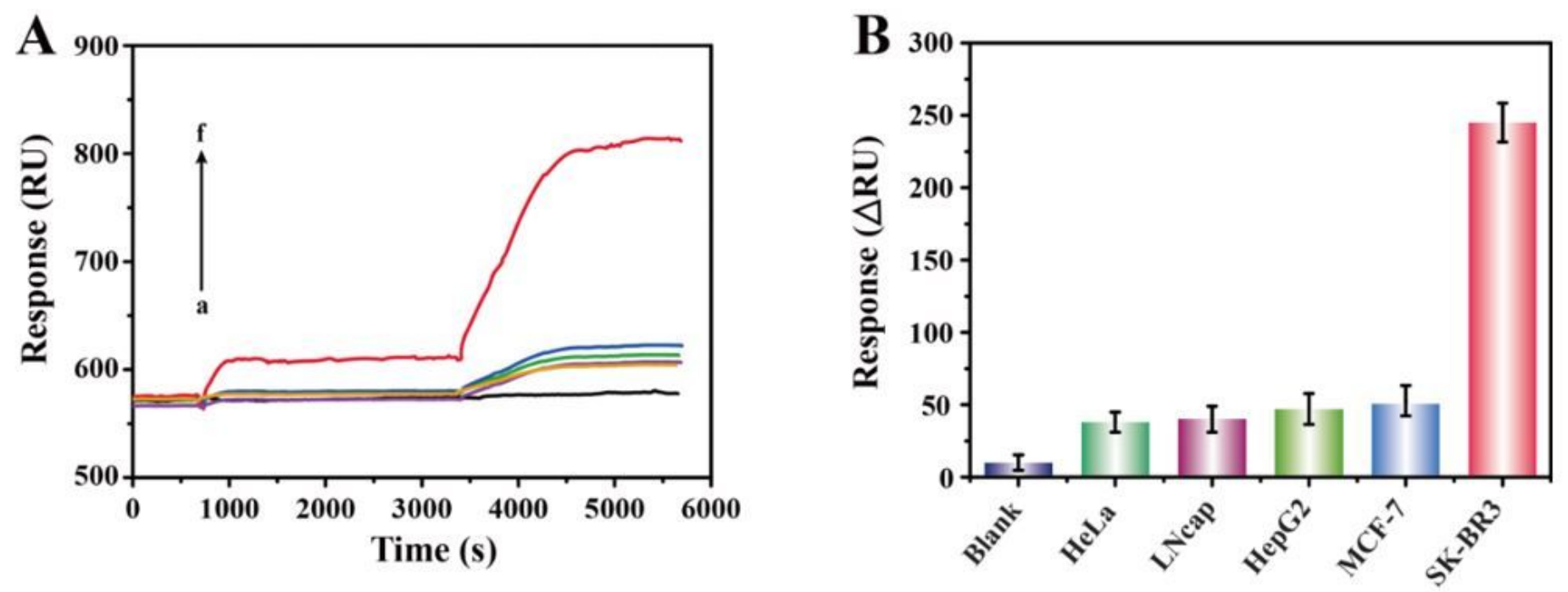

Figure 4

Evaluation of the specificity of the sensing strategy. (A) SPR detection sensorgrams and (B) the response signals of (a) blank control and exosomes (1.0 × 107 particles/mL) derived from (b) HeLa, (c) LNcap, (d) HepG2, (e) MCF-7, and (f) SK-BR3 cell lines, respectively. All data expressed as mean \pm standard variation $(n=3)$. 

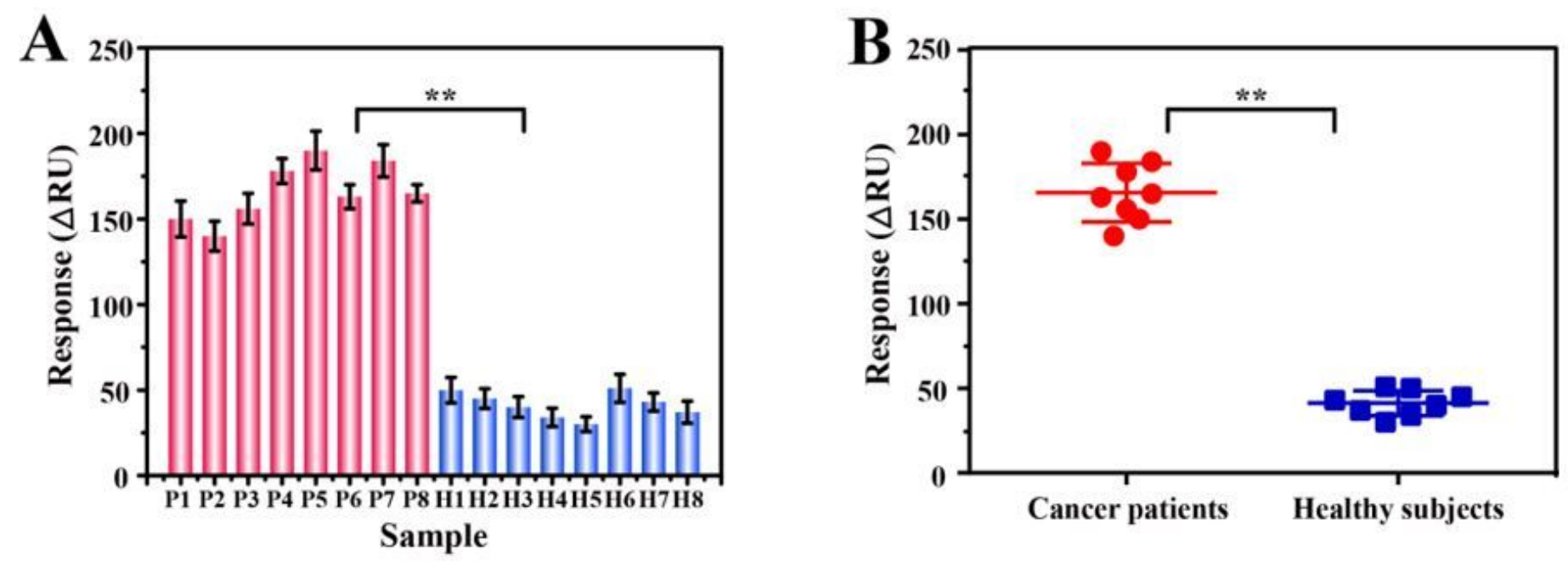

Figure 5

Investigation of the clinical applicability of this biosensor. (A) Analysis of exosomes from clinical samples (breast cancer patients: P1 P8 and healthy control subjects: H1 H8) by the developed strategy and (B) the corresponding scatter plot. Significance was determined by Student unpaired t-test, $* * p<$ 0.05 .

\section{Supplementary Files}

This is a list of supplementary files associated with this preprint. Click to download.

- scheme1.jpg

- SupplementaryMaterial.docx 